\title{
PRIMARY IMPLANT STABILITY: A LEAP TOWARDS SUCCESSFUL OSSEOINTEGRATION - A NARRATIVE REVIEW
}

\section{Dr. G. Kishore Kumar, Dr. Shanmuga Priya, Dr. U. Arunmozhi, Dr. R. Kadhiresan, Dr. R. A. Jenifer Cynthia}

Department of Periodontology and Oral Implantology, Sri Venkateswara Dental College and Hospital, Off OMR road, Near Navalur, Thalambur, Chennai-603103

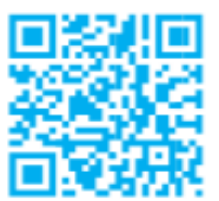

\section{DOI: 10.37841/jidam_2021_V8_14_03}

Address for Correspondence:

Dr. G. Kishore Kumar,

Post Graduate Student,

Department of Periodontology and Oral Implantology

Sri Venkateswara Dental College and Hospital

Off OMR road, Near Navalur, Thalambur, Chennai-603103

E-mail: kishorekk56743@gmail.com

Received: 24.08.2021

First Published: 28.11.2021

Accepted: 20.11.2021

Published: 27.12.2021

\begin{abstract}
Implant stability can be defined as an absence of clinical implant mobility and consists of primary and secondary implant stability. It has been recognised as one of the most important and useful factors when it comes to predicting implant anchorage. Primary stability includes the mechanical attachment of an implant in the surrounding bone at the insertion, whereas secondary implant stability is the tissue response to the implant and subsequent bone remodelling processes. It is known to be a crucial factor for successful osseointegration of dental implants. There is sufficient evidence to accept a positive correlation between primary implant stability and implant success, as the success relies on the sustainable integration of the implants into hard and soft tissues. This review extensively focuses on different factors affecting primary stability such as implant design and characteristics, bone quality and methods to measure implant stability.
\end{abstract}

KEYWORDS: bone density, dental implants, implant stability, osseodensification, osseointegration. 


\section{INTRODUCTION}

Dental implants are highly sort treatment options for reconstructing and rehabilitating partial or completely edentulous jaws. Literature shows dental implants have been highly successful treatment modality for several decades. Patients seeking dental implants for restoration of lost tooth have become higher in the past 30 years. The number of implants placed every year is increasing exponentially because the cumulative mean implant success rate is $89.7 \% \pm 10.2 \%$ after a mean post functional loading period of 15.7 years ${ }^{1}$.

But Failure is inevitable in any surgical situation, and dental implants are not an exception. Dental implant failure can be broadly divided into early and late failures. Early implant failure occurs usually within first three or months after the placement of implants and before the insertion of abutment or the crown. This undoubtedly affects the primary implant stability. Late implant failures are expected to occur after the prosthetic fixtures have been placed and the implant have been loaded for a period of time. The implant failure rates vary.1.2 to $3 \%$ fail during early stages and 0 to $1.8 \%$ during late stages. It appears that early failure is more common than late failures ${ }^{2}$.

One of the most important factors in preventing early implant failure is maintenance of Implant stability. Implant stability mainly depends on the design of the implant (micro and macro), surgical technique used for the placement of implants and the bone (Quality and quantity). This narrative review discusses on various factors influencing the primary implant stability, and the techniques and factors to enhance the same ${ }^{3}$.

\section{IMPLANT STABILITY}

Osseointegration is the direct structural and functional connection between living bone and the surface of a load carrying implant. It is a quite complex process which involves continuous bone apposition and remodelling at the bone-implant interface. For the success of osseointegration implant stability which is a prerequisite. Implant stability occurs in two stages: primary and secondary.

\section{- PRIMARY STABILITY:}

Primary stability refers to the initial mechanical stability at the time of implant placement. Initial stability of an implant is due to the mechanical anchorage into the surrounding bone; it is assessed by the contact area between the body of the implant and the bony site. When there is micro motion present around the implant, it not only compromises the primary stability of the implant but also impairs the osteointegration of the implant.

\section{- SECONDARY STABILITY:}

Secondary stability is a biological form of stability through bone regeneration and remodelling at the implant-tissue interface. It depends on the implant healing process, implant surface condition and also primary implant stability. Total implant stability is a measure of both primary and secondary implant stability.

\section{FACTORS THAT AFFECT PRIMARY IMPLANT STABILITY:}

Various studies have been performed to assess factors which contribute to primary implant stability. (Fig 1)

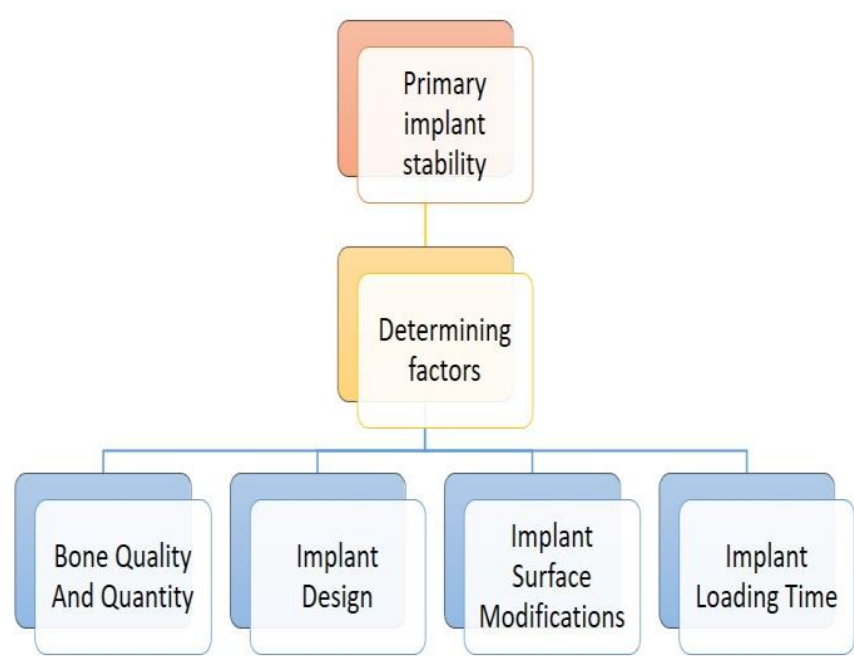

Fig 1:Factors affecting Primary Stability

\section{BONE QUALITY AND QUANTITY:}

A strong correlation exists between primary implant stability and bone density. Higher implant stability is found in dense cortical bone (type I) of mandible than in porous cancellous bone (type IV) of maxilla as inferred by the following studies.

In an in-vitro study by Kashi et al, implants were placed in polyurethane composite bone blocks of different bone quality as classified by Carl Misch. The success of an implant depends on the ISQ (Implant stability quotient) value. ISQ value of 60-70 indicates better implant stability and values less than 45 indicates poor stability. They found that higher implant stability values of ISQ in Type II bone (55.23 - 62.1) than in Type IV bone of (52.03-54.38) ${ }^{5}$. 
Stefan rues et al, in an invitro study assessed the effect of bone quality and quantity on the primary stability of dental implants in a pig scapulae using micro-computed tomography. They concluded that primary implant stability was not dependent on the total bone thickness but correlated with increasing bone mineral density or overall cortical bone thickness ${ }^{6}$.

\section{IMPLANT DESIGN:}

Implant design modifications have been made for achieving better implant stability. The tapered dental implants have replaced the cylindrical and parallel designs. These tapered implants are highly esthetic and produces a degree of compression of cortical bone in deficient sites which increases primary stability.

Markovic et al compared self-tapping and non-self-tapping implants and showed that both type of implants had increased initial primary stability but higher stability was seen in self-tapping implants ${ }^{7}$.

With different thread depths and at the cost of increased thread exposure analysed their clinical outcomes over a one-year period. They concluded that, given the same bone quality and the same bone site preparation, implant insertion is easier when using a reduced thread depth while implants with a larger thread diameter need higher insertion torque values to reach greater primary stability ${ }^{8}$.

\section{IMPLANT SURFACE MODIFICATION}

Primary stability of the implant also depends upon the surface characteristics of the Implant. These changes can be brought by an additive surface treatment (Titanium plasma spraying, Hydroxy apatite coated surface), Subtractive surface treatment (Blasting with titanium oxide/aluminium oxide, acid etched surface) or modified surface treatment (Laser induced roughened surfaces, Ion implantation and oxidized surface treatments). All these modifications bring roughened implant surface which have a large surface area that provides a better bone implant interface than a machined implant.

Franchi et al conducted a study to evaluate the effect of acid etched and sand blasted implant surface treatment on osteointegration. The histomorphometric analysis of bone to implant contact (BIC), bone ingrowth and bone surface showed that acid etched and sandblasted implant surface promoted osteointegration by increasing the growth and metabolic activity of osteoblast. Their study showed that the osteocyte acted as a microscopic scaffold for mesenchymal and osteoblast like cell adhesion. This enhances periimplant osteogenesis ${ }^{9}$.

Recently a study conducted by Fei Sun et al evaluated the effect of alkali storage solution on SLA treated implants. Among the three study groups (Group 1 sandblasting acid etching (SLA), group 2 oxidation (SLA which is oxidised) and group 3 storage groups (SLA samples that were oxidized and stored in $0.1 \mathrm{~mol} / \mathrm{L} \mathrm{NaOH}$ solution) SLA implants stored in alkali medium maintained the surface characteristics of plasma oxidation showed better primary stability than other groups ${ }^{10}$.

\section{LOADING TIME}

Simunek et al evaluated the gain in stability of implants placed in different quality of bone after immediate loading. Based on primary stability the study groups were divided into three group L Low (ISQ 68), group M Medium (ISQ 68 to72), and group H High (ISQ 72). After 10 weeks it was noted that implants with low and medium primary stability showed a significant gain in RFA values, whereas on the contrary the higher group which had a good stability earlier saw a decline in RFA values over time ${ }^{11}$.

Many studies on the effect of loading time and primary stability have inferred that lower stability values were seen in one staged and immediately loaded implants but the stability increases after 3 months because of bone healing and remodeling.

\section{METHODS TO IMPROVE PRIMARY IMPLANT STABILTY}

Implant stability can be enhanced by optimum preparation of the implant bed. Several techniques have been proposed (Fig 2) 


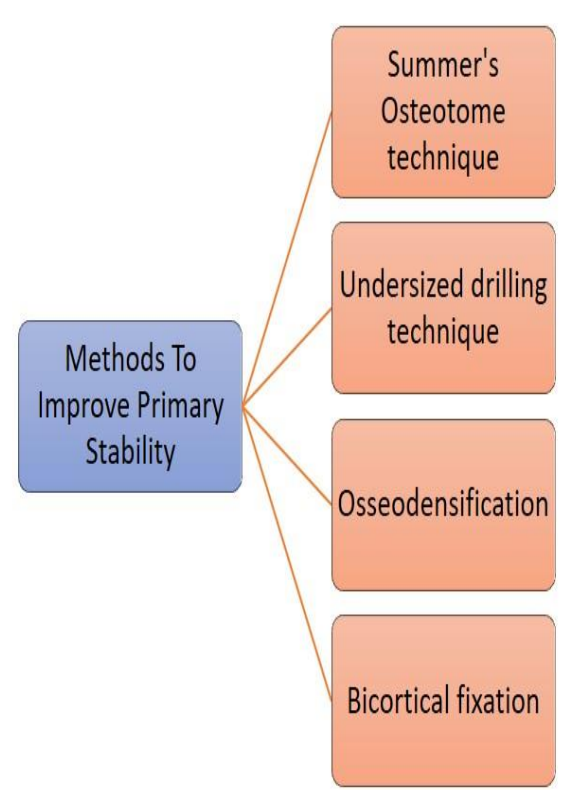

Fig 2: Methods to Improve Primary Implant Stability

\section{Summers Osteotome Technique}

It uses lateral bone condensation technique where a series of osteotomes were tapped into the implant site after the pilot drill. This lateral compaction results in denser bone and thus provides better primary implant stability than conventional technique

\section{Under Sizing Drilling Technique}

Maximum bone volume is preserved and bone density is enhanced by undersize drilling technique the implants are inserted using smaller diameter drills in the underprepared osteotomy site, which aids in translocation of osteogenic bone fragments on the implant surface and promotes bone healing and remodelling. Fewer studies state that $10 \%$ undersized preparation provides additional stability to the implants whereas in a recent systematic review, it was concluded that undersized drilling is effective in increasing implant insertion torque and implants inserted with $26 \%$ undersized protocol reached significantly higher insertion torque.

Jayant et al (2020), in his systematic review compared undersized drilling and conventional drilling technique. The use of undersize drilling was more advantageous in increasing initial implant stability in the low-density bone. Although the Primary Implant Stability may be lower in conventional drilling technique, the secondary implant stability was found to be increased ${ }^{12}$.

\section{Osseodensification technique:}

Similar to traditional osteotome technique Osseodensification simultaneously compacts and autografts the bone tissue in an outward expanding direction without creating trauma. The main feature of the osseodensification burs is it rotates in counter clockwise direction and in a noncutting motion thereby condensing the bone along the walls of the implant bed.

In a systematic review by N.M. Padhye et al., they analysed 12 studies and claimed that osseodensification method increased the insertion torque from $25 \mathrm{Ncm}$ for implants placed using standard drilling technique to $49 \mathrm{Ncm}$ in low density bone. It was also noted that if osseodensification attributed to the viscoelastic nature of the bone causing spring back effect of the bone creating compressive forces $^{13}$.

Inchigolo A.D., (2021) in his recent systematic review resulted in $19.4 \%$ higher bone implant contact than conventional and Summer's Osteotome technique ${ }^{14}$.

\section{Bicortical fixation:}

This technique uses longer implants to engage 2 layers of cortical bone. It includes cervical crest and the sinus or nasal floor cortex or lower border of anterior mandibular cortex. Though it is a simpler and economical technique, it is not used nowadays because primary and secondary implant stability of bicortical fixation was not more superior when compared to unicortical fixation and indirect sinus elevation.

\section{METHODS TO MANAGE IMPLANTS WITH LOW OR NO PRIMARY IMPLANT STABILITY}

- If the primary implant stability is not achieved by the planned implant, a longer or a wider implant with aggressive thread deign can be used to engage bone and enhance the stability

- Addition of bone grafts to the jumping distance of the immediate implants provides wedging effect to the implant and prevents any micro motion during healing

- In soft trabecular bone, submerged implants with primary closure of the flap were preferred along with prolonged healing time to avoid micromotion of the implant and promote adequate osseointegration. 
- The implant placement should be aborted if the above-mentioned methods cannot attain a stable implant in a prosthetically driven position ${ }^{3}$.

\section{METHODS TO MEASURE IMPLANT STABLITY}

Objective measurement of implant stability helps the surgeon to decide about the protocol and the treatment methods for each case. There are various methods to evaluate implant stability ${ }^{4}$. They are (Fig 3)

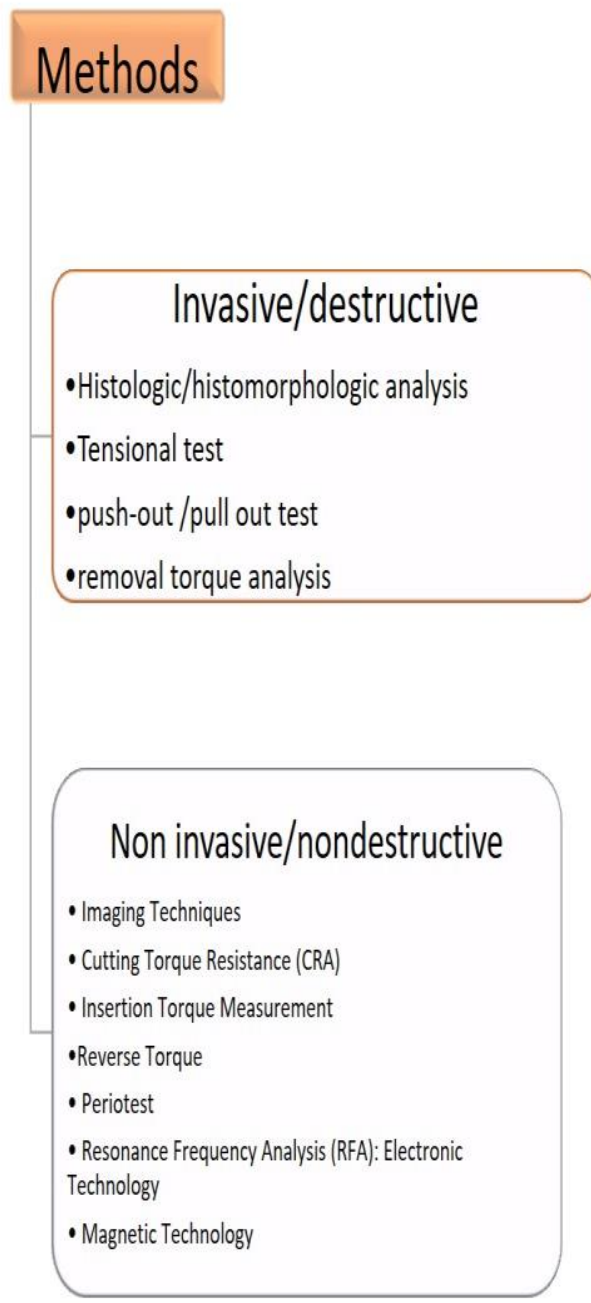

Fig 3: Methods to Measure Primary Implant Stability

\section{INVASIVE/ DESTRUCTIVE METHODS}

\section{Histologic/ Histomorphologic analysis}

It is used to measure the bone implant contact (BIC) and peri-implant bone quantity using dyes around implant, though it gives us accurate measurements it is not followed because of its invasiveness ${ }^{15}$.

\section{Tensional test}

Tensional test was earlier assessed by detaching the implant from the supporting bone. It was later modified by Branemark by applying the lateral load to the implant fixture. However, this method is not pursued because of the difficulties in translating the test results to any area independent of mechanical properties ${ }^{16}$.

\section{Push-out/pull-out test}

This test investigates the healing capabilities at the bone implant interface. It measures interfacial shear strength by applying load parallel to implant-bone interface which is assessed during the healing period. As it is technique sensitive it can be Applicable to only non-threaded implants ${ }^{17}$.

\section{Removal torque analysis}

In removal torque analysis, when the reverse or unscrewing torque is more than $20 \mathrm{Ncm}$ it is considered as stable implant but the major disadvantage in this technique is the fracture of abutment connection with the implant surface. So, this test is limited only on preclinical applications ${ }^{18}$.

\section{NON-INVASIVE/NON-DESTRUCTIVE METHODS FOR ASSESSING IMPLANT STABILITY}

\section{Imaging techniques}

Imaging techniques are used to assess the health of the implant by evaluating the bone quantity and quality changes. Computer assisted measurement of crestal bone level change are proved to be the most accurate radiographical information. The main advantage of Cone beam CT is that it allows us to measure bone implant contact and bone density around the implants immediately after placement ${ }^{19}$.

\section{Cutting torque resistance analysis (CRA)}

It is measured during low-speed drilling by controlling the hand pressure. It measures the amount of unit volume of bone removed by an electric motor. CRA gives a more objective assessment of bone density but the bone quality information cannot be assessed until the osteotomy site is prepared $^{20}$.

\section{Insertion torque measurement}

The most common method of assessing the bone quality during implant placement is the insertion torque values. It is an independent measurement for primary stability but it 
cannot assess the secondary stability by new bone formation. An increase in insertion torque may highlight an increase in primary stability ${ }^{21}$.

\section{Reverse torque test}

To evaluate the secondary stability of the implant Roberts et al proposed reverse torque test. Implants that rotate when reverse torque is applied indicate that Bone Implant Contact (BIC) could be destroyed. If this test is performed it might itself lead to the failure of the implant because of the stress applied during torquing ${ }^{22}$.

\section{Periotest}

Periotest measure the mobility of an implant to a defined implant load by assessing the reaction of peri-implant tissue indeed periotest was primarily introduces to measure the damping characteristics of periodontal ligament thereby assessing the mobility of natural tooth it has an electronically controlled and electromagnetically driven metallic rod in an hand piece. Periotest value range from -8 (low mobility) to +50 (high mobility) ${ }^{23}$.

\section{Resonance frequency analysis}

RFA measures implant stability and bone density at various time points using vibration and a principle of structural analysis. It starts with a low frequency and increases the pitch until the implant starts resonating. Stronger bone implant interface is indicated by high frequency resonance. Currently, Wireless RFA gadgets have been developed.

\section{Electronic Technology Resonance Frequency Analysis (Osstell'TM)}

Osstell was the first commercially available device for measuring implant stability it combine the transducer, computerised analysis and the excitation source into one machine. It translates the resonance frequency ranging from 3000 to $8500 \mathrm{~Hz}$ as the implant stability quotient $0-100$ which is the measurement unit used in this device ${ }^{24}$.

\section{Magnetic Technology Resonance Frequency Analysis (Osstell ${ }^{\text {TM }}$ Mentor)}

The transducer has a magnetic peg which is activated by magnetic resonance frequency probe. It vibrate sand induces electric volt sampled by the magnetic analyser and the values are expressed in terms of ISQ 0-100. But this method is technique sensitive and expensive and requires high clinical skill otherwise the measured values will be affected $^{25}$.

\section{CONCLUSION:}

For successful osseointegration, the micromotion of dental implants should not be beyond $50-100 \mu$ during implant placement. This narrative review emphasizes beyond doubt that primary stability is one of the cardinal factors to determine the longterm stability.

Though higher primary stability is attained in D1 cortical bone when compared to D3 or D4 cancellous bone, clinical success of implants in compromised sites is achievable by adapting to novel techniques and implants designs. Symmetrical, spiral-shaped threads, and micro rings at the cervical part of implants are being preferred as it enhances contact with surrounding cortical plate. Oxidised sandblasted - SLA implants increased the bone implant surface contact by encouraging migration of osteocyte to the implant surface.

Thus the present concept of Osseodensification is a promising alternative to conventional technique in compromised sites. It overcomes the complexity of various other treatment modalities available to achieve primary stability of implants.

\section{FINANCIAL SUPPORT AND SPONSORSHIP}

Nil

\section{CONFLICTS OF INTEREST}

There are no conflicts of interest.

\section{REFERNCES:}

1. $\mathrm{Fu} \mathrm{J}-\mathrm{H}$, Wang H-L. Breaking the wave of peri-implantitis. Periodontol 2000. 2020;84:145-160.

2. Olmedo-Gaya MV, Manzano-Moreno FJ, CañaveralCavero E, de Dios Luna-del Castillo J, VallecilloCapilla M. Risk factors associated with early implant failure: A 5-year retrospective clinical study. J Prosthet Dent. 2016 Feb;115(2):150-5.

3. shadid RM, Sadaqah NR, Othman SA. Does the Implant Surgical Technique Affect the Primary and/or Secondary Stability of Dental Implants? A Systematic Review. Int J Dent. 2014;2014:204838

4. Swami V, Vijayaraghavan V, Swami V. Current trends to measure implant stability. J Indian Prosthodont Soc. 2016 Apr-Jun;16(2):124-30. doi: 10.4103/09724052.176539. PMID: 27141160; PMCID: PMC4837777. 
5. Kashi A, Gupta B, Malmstrom H, Romanos GE. Primary stability of implants placed at different angulations in artificial bone. Implant Dent 2015;24:92-5

6. Rues S, Schmitter M, Kappel S, Sonntag R, Kretzer JP, Nadorf J. Effect of bone quality and quantity on the primary stability of dental implants in a simulated bicortical placement. Clin Oral Investig. 2021 Mar;25(3):1265-1272. doi: 10.1007/s00784-02003432-z. Epub 2020 Jul 10. PMID: 32651646; PMCID: PMC7878229

7. Marković A, Calvo-Guirado JL, Lazić Z, Gómez-Moreno G, Ćalasan D, Guardia J, et al. Evaluation of primary stability of self-tapping and nonself-tapping dental implants. A 12-week clinical study. Clin Implant Dent Relat Res 2013;15:341-9

8. Menini M, Bagnasco F, Calimodio I, Di Tullio N, Delucchi F, Baldi D, Pera F. Influence of Implant Thread Morphology on Primary Stability: A Prospective Clinical Study. Biomed Res Int. 2020 Aug 5;2020:6974050. doi: 10.1155/2020/6974050. PMID: 32802868; PMCID: PMC7426766

9. Franchi, M., Bacchelli, B., Giavaresi, G., De Pasquale, V., Martini, D., Fini, M., Giardino, R. and Ruggeri, A. Influence of Different Implant Surfaces on PeriImplant Osteogenesis: Histomorphometric Analysis in Sheep. Journal of Periodontology, (2007), 78: 879888. https://doi.org/10.1902/jop.2007.060280.

10. Sun, Fei, Shao-Jie Li, Xin-Chang Li, Lei Wang, DeChun Ba, Gui-Qiu Song, Chuan-Sheng Sun, and Zeng Lin.. "Effect of Surface Modification on the Primary Stability of Dental Implants by Plasma Oxidation and Storage Treatment" Coatings 10, no. 2020 7: 622.

11. Simunek A, Kopecka D, Brazda T, Strnad I, Capek L, Slezak R. Development of implant stability during early healing of immediately loaded implants. Int J Oral Maxillofac Implants. 2012 May-Jun;27(3):619-27. PMID: 22616056

12. Palaskar JN, Joshi N, Shah PM, Gullapalli P, Vinay V. Influence of different implant placement techniques to improve primary implant stability in low-density bone: A systematic review. J Indian Prosthodont Soc 2020;20:11-6

13. Padhye NM, Padhye AM, Bhatavadekar NB. Osseodensification -- A systematic review and qualitative analysis of published literature. J Oral Biol Craniofac Res. 2020 Jan-Mar;10(1):375-380. doi: 10.1016/j.jobcr.2019.10.002. Epub 2019 Nov 2. PMID: 31737477 ; PMCID: PMC6849357.
14. Inchingolo AD, Inchingolo AM, Bordea IR, Xhajanka E, Romeo DM, Romeo M, Zappone CMF, Malcangi G, Scarano A, Lorusso F, Isacco CG, Marinelli G, Contaldo M, Ballini A, Inchingolo F, Dipalma G. The Effectiveness of Osseodensification Drilling Protocol for Implant Site Osteotomy: A Systematic Review of the Literature and Meta-Analysis. Materials (Basel). 2021 Feb 28;14(5):1147. doi: 10.3390/ma14051147. PMID: 33671038; PMCID: PMC7957527.

15. Nkenke E, Hahn M, Weinzierl K, Radespiel-Tröger M, Neukam FW, Engelke K. Implant stability and histomorphometry: A correlation study in human cadavers using stepped cylinder implants. Clin Oral Implants Res 2003;14:601-9

16. Meenakshi S, Raghunath N, Raju SN, Srividya S, Indira PN. Implant stability a key determinant in implant integration. Trends Prosthodont Dent Implantol 2013;4:28-48.

17. Brunski JB, Puleo DA, Nanci A. Biomaterials and biomechanics of oral and maxillofacial implants: Current status and future developments. Int $\mathrm{J}$ Oral Maxillofac Implants 2000;15:15-46.

18. Sullivan DY, Sherwood RL, Collins TA, Krogh PH. The reverse-torque test: A clinical report. Int $\mathrm{J}$ Oral Maxillofac Implants 1996;11:179-85

19. Wyatt CC, Pharoah MJ. Imaging techniques and image interpretation for dental implant treatment. Int $\mathbf{J}$ Prosthodont 1998;11:442-52

20. Johansson P, Strid K. Assessment of bone quality from cutting resistance during implant surgery. Int $\mathrm{J}$ Oral Maxillofac Implants 1994;9:279-88.

21. Bayarchimeg D, Namgoong H, Kim BK, Kim MD, Kim S, Kim TI, et al. Evaluation of the correlation between insertion torque and primary stability of dental implants using a block bone test. J Periodontal Implant Sci 2013;43:30-6

22. O'Sullivan D, Sennerby L, Jagger D, Meredith N. A comparison of two methods of enhancing implant primary stability. Clin Implant Dent Relat Res 2004;6:48-57

23. Schulte W, d'Hoedt B, Lukas D, Muhlbradt L, Scholz F, Bretschi J, et al. Periotest - A new measurement process for periodontal function. Zahnarztl Mitt 1983;73:1229-30, 1233-6, 1239-40

24. Valderrama P, Oates TW, JonesAA, Simpson J, Schoolfield JD, Cochran DL. Evaluation of two different resonance frequency devices to detect implant stability: A clinical trial. J Periodontol 2007;78:262-72. 
Kishore et al: Primary Implant Stability: A Leap towards Successful Osseointegration

25. Park JC, Lee JW, Kim SM, Lee JH. Implant Stability Measuring Devices and Randomized Clinical Trial for ISQ Value Change Pattern Measured from Two Different Directions by Magnetic RFA, Rapidly Evolving Practice 2011;5:111-30 